\title{
Signals of Warped Extra Dimensions at the LHC
}

\author{
P. Osland ${ }^{*}$, A. A. Pankov ${ }^{\dagger}$, A. V. Tsytrinov ${ }^{\dagger}$ and N. Paver** \\ *Department of Physics and Technology, University of Bergen, N-5020 Bergen, Norway \\ ${ }^{\dagger}$ The Abdus Salam ICTP Affiliated Centre, Technical University of Gomel, 246746 Gomel, Belarus \\ ${ }^{* *}$ University of Trieste and INFN-Trieste Section, 34100 Trieste, Italy
}

\begin{abstract}
We discuss the signatures of the spin-2 graviton excitations predicted by the RandallSundrum model with one warped extra dimension, in dilepton and diphoton production at LHC. By using a specific angular analysis, we assess the ranges in mass and coupling constant where such gravitons can be discriminated against competitor spin- 1 and spin-0 objects, that potentially could manifest themselves in these processes with the same mass and rate of events. Depending on the value of the coupling constant to quarks and leptons, the numerical results indicate graviton identification mass ranges up to $1.1-2.4 \mathrm{TeV}$ and $1.6-3.2 \mathrm{TeV}$ for LHC nominal energy of $14 \mathrm{TeV}$ and time-integrated luminosity of 10 and $100 \mathrm{fb}^{-1}$, respectively.
\end{abstract}

Keywords: LHC, graviton, extra dimensions, spin

PACS: $12.60 .-\mathrm{i}, 11.10 . \mathrm{Kk}, 12.60 . \mathrm{Cn}$

\section{INTRODUCTION}

Originally, extra spatial dimensions were proposed to address the mass scale hierarchies affecting the Standard Model (SM), and requiring parameter fine tuning, in particular the so-called gauge hierarchy $M_{\mathrm{EW}} \ll M_{\mathrm{Pl}} \sim 10^{16} \mathrm{TeV}$. These scenarios predict the existence of heavy new particles, or excitations of the SM particles, that could be revealed as narrow peaks in cross sections measured at the LHC if their masses were within the experimental kinematical reach. Observability will depend on the values of these heavy 'resonance' masses and coupling constants to the SM matter, that assure sufficiently high event rates.

Actually, in this regard, the discovery reach on an individual scenario is the maximum value of the corresponding resonance mass, $M_{R}$, for which the peak can be observed. However, different non-standard models can give, for appropriate values of their parameters, peaks at the same $M=M_{R}$ and the same number of events under the peak. Accordingly, one defines for any non-standard scenario an identification mass range (of course, included within the discovery range), where it can be unambiguously discriminated as the one really underlying the peak, by excluding the other models as potential sources of that same peak. The determination of the spin of a discovered heavy resonance is, therefore, crucial for its identification. We will here discuss the identification of the spin-2 graviton excitation predicted by the Randall-Sundrum (RS) model with one warped extra dimension [1], against the spin-1 and the spin-0 hypotheses for a heavy neutral resonance discovered in dilepton and diphoton inclusive production at the LHC:

$$
p+p \rightarrow l^{+} l^{-}+X \quad(l=e, \mu) \text { and } p+p \rightarrow \gamma \gamma+X
$$


We will model the spin- 1 hypothesis by the $Z^{\prime}$ s predicted by extended electroweak gauge symmetries [2], and the spin- 0 one by the sneutrinos $(\tilde{v})$ envisaged by $R$-parity violating SUSY extensions of the SM [3]. 'Confusion' domains of their respective parameter spaces allowed by current experimental constraints exist, in which ' $s$-channel' exchanges of the above mentioned particles can in the process (1) produce narrow peaks in the dilepton invariant mass with the same values of mass and event rates. Therefore, for the spin-2 hypothesis discrimination against the two alternative ones, more detailed information must be supplied, i.e., that embodied in the characteristic angular distributions of the different scenarios.

\section{BASIC OBSERVABLES AND ANGULAR ANALYSIS}

The basic observable for a heavy resonance discovery at an invariant dilepton (or diphoton) mass $M=M_{R}$ (with in our case $R=G, Z^{\prime}, \tilde{v}$ denoting graviton, $Z^{\prime}$ and sneutrino, respectively) is the production cross section governing the rate of events

$$
\sigma(p p \rightarrow R) \cdot \operatorname{BR}\left(R \rightarrow l^{+} l^{-}\right)=\int_{-z_{\text {cut }}}^{z_{\mathrm{cut}}} \mathrm{d} z \int_{M_{R}-\Delta M / 2}^{M_{R}+\Delta M / 2} \mathrm{~d} M \int_{y_{\min }}^{y_{\max }} \mathrm{d} y \frac{\mathrm{d} \sigma}{\mathrm{d} M \mathrm{~d} y \mathrm{~d} z},
$$

and the differential angular distribution

$$
\frac{\mathrm{d} \sigma}{\mathrm{d} z}=\int_{M_{R}-\Delta M / 2}^{M_{R}+\Delta M / 2} \mathrm{~d} M \int_{y_{\min }}^{y_{\max }} \frac{\mathrm{d} \sigma}{\mathrm{d} M \mathrm{~d} y \mathrm{~d} z} \mathrm{~d} y .
$$

In Eqs. (2) and (3), $z=\cos \theta_{\mathrm{cm}}$ and $y$ define the lepton-quark (or photon-quark) angle in the dilepton (or diphoton) center-of-mass and the dilepton rapidity, respectively, and cuts on phase space due to detector acceptance are indicated. For integration over the full phase space, the limits would be $z_{\text {cut }}=1$ and $y_{\max }=-y_{\min }=\log (\sqrt{s} / M)$ with $\sqrt{s}$ the LHC collider center-of-mass energy. Furthermore, $\Delta M$ is an invariant mass bin around $M_{R}$, reflecting the detector energy resolution, see for instance Ref. [4]. To evaluate the number $N_{S}$ of resonant signal events, these equations must be multiplied by the timeintegrated luminosity, for which we will take 100 and $10 \mathrm{fb}^{-1}$, and by the foreseen reconstruction efficiencies (90\% for both electrons and muons [5]). Also, the typical experimental cuts on rapidities and transverse momenta must be applied $\left(p_{\perp}>20 \mathrm{GeV}\right.$ and $|\eta|<2.5$ for both leptons). Finally, with $N_{B}$ the number of 'background' events in the $\Delta M$ bin, determined by the SM predictions, the criterion $N_{S}=5 \sqrt{N_{B}}$ or 10 events, whichever is larger, will be adopted as the minimum signal for the peak discovery. Of course, the determination of discovery and identification reaches on the different nonstandard models requires the expression of Eqs. (2) and (3) in terms of convolutions of the pertinent partonic cross sections times parton distribution functions, and for the latter we will choose the CTEQ6 ones of Ref. [6].

Since the $z$-dependence of the cross sections for graviton, $Z^{\prime}$ and sneutrino exchanges are different, the discrimination of spin- 2 from spin- 1 and spin- 0 might be attempted by the 'direct' comparison of Eq. (3) for the three hypotheses [7, 8].

In practice, due to the completely symmetric $p p$ initial state at the LHC, the determination of the sign of $z$ from the measured events may not be fully unambiguous. An 
observable potentially avoiding this ambiguity, and which we adopt to perform the angular analysis, is the z-evenly integrated center-edge angular asymmetry $[9,10]$ :

$$
A_{\mathrm{CE}}=\frac{\sigma_{\mathrm{CE}}}{\sigma} \quad \text { with } \quad \sigma_{\mathrm{CE}} \equiv\left[\int_{-z^{*}}^{z^{*}}-\left(\int_{-z_{\text {cut }}}^{-z^{*}}+\int_{z^{*}}^{z_{\mathrm{cut}}}\right)\right] \frac{\mathrm{d} \sigma}{\mathrm{d} z} \mathrm{~d} z .
$$

In Eq. (4), $0<z^{*}<z_{\text {cut }}$ defines the separation between the "center" and the "edge" angular regions, and is a priori arbitrary. However, its actual value can be "optimized" in the numerical analysis. A further potential advantage of $A_{\mathrm{CE}}$ is that, as consisting of ratios of integrated cross sections, it could be less sensitive to systematic uncertainties, such as those stemming from particular choices of parton distributions, $K$-factor values, etc. ${ }^{1}$

\section{NON-STANDARD MODELS AND ANGULAR DISTRIBUTIONS}

\section{RS model with one compactified warped extra dimension}

This simplest version consists of one warped extra dimension, $y$, two three-dimensional branes placed at a compactification relative distance $\pi R_{c}$ in $y$, and the specific 5-D metric [1]

$$
d s^{2}=\exp (-2 k|y|) \eta_{\mu v} d x^{\mu} d x^{v}-d y^{2}
$$

where $\eta_{\mu \nu}$ is the usual Minkowski tensor and $k>0$ is a dimensionful constant. The $\mathrm{SM}$ fields are assumed to be localized to the so-called TeV brane, while gravity can propagate in the full 5-D space, in particular on the other brane, the Planck brane, in which the effective 4-D mass scale is $\bar{M}_{\mathrm{Pl}}=1 / \sqrt{8 \pi G_{\mathrm{N}}}=2.44 \times 10^{15} \mathrm{TeV}$. With $M_{*}$ the 5-D mass scale, analogously related to the cubic root of the 5-D gravitational constant, Einstein's equations imply the relation $\bar{M}_{\mathrm{Pl}}^{2}=\left(M_{*}{ }^{3} / k\right)\left(1-\exp \left(-2 k \pi R_{c}\right)\right)$, and the basic 'naturalness' assumption imposed on the model, to avoid further fine tunings, is $\bar{M}_{\mathrm{Pl}} \sim M_{*} \sim k$. The geometry of Eq. (5) implies that the mass spectrum on the Planck brane, of the order of $10^{15} \mathrm{TeV}$, can for $k R_{c} \sim 11$ be exponentially 'warped' down to the $\mathrm{TeV}$ brane where SM particles live, by many orders of magnitude, namely, to the scale $\Lambda_{\pi}=\bar{M}_{\mathrm{Pl}} \exp \left(-k \pi R_{c}\right) \sim 1 \mathrm{TeV}$. The appealing consequence is then that gravitational effects can occur in the reach of supercolliders, such as the LHC. Indeed, junction conditions at the brane $y$-positions imply, for the above value of $k R_{c}$, the existence of a tower of spin-2 graviton excitations, $h_{\mu \nu}^{(n)}$, with a specifically spaced mass spectrum $M_{n}=x_{n} k \exp \left(-k \pi R_{c}\right)$ of order $\mathrm{TeV}\left(x_{n}\right.$ are here the roots of $\left.J_{1}\left(x_{n}\right)=0\right)$. Their couplings to the SM particles are only $1 / \Lambda_{\pi}$-suppressed (not $1 / \bar{M}_{\mathrm{Pl}}$ ):

$$
\mathscr{L}_{\mathrm{TeV}}=-\left[\frac{1}{\bar{M}_{\mathrm{Pl}}} h_{\mu v}^{(0)}(x)+\frac{1}{\Lambda_{\pi}} \sum_{n=1}^{\infty} h_{\mu v}^{(n)}(x)\right] T^{\mu v}(x),
$$

\footnotetext{
${ }^{1}$ Recently, an asymmetry $\tilde{A}_{\mathrm{CE}}$ defined along these lines, in terms of differences between lepton and antilepton pseudorapidities, has been proposed for model identification in Ref. [11]. Also, the possibility of identifying the graviton excitation spin- 2 from the azimuthal angular dependence of the graviton + jet inclusive production has been explored in [12].
} 
and their signature may appear at LHC. In (6), $T^{\mu v}$ is the energy-momentum tensor and $h_{\mu \nu}^{(0)}$ denotes the zero-mode, ordinary, graviton.

The commonly chosen independent RS model parameters are the mass of the lowest graviton excitation, $M_{G} \equiv M_{1}$, and the 'universal', dimensionless, coupling $c=k / \bar{M}_{\mathrm{Pl}}$ (the scale $\Lambda_{\pi}$ is then a derived quantity). Theoretically 'natural' ranges of these parameters are $0.01 \leq c \leq 0.1$ and $\Lambda_{\pi}<10 \mathrm{TeV}$ [13]. With $\Gamma_{n}=\rho M_{n} x_{n}^{2} c^{2}$ and $\rho$ a number of the order of 0.1 , narrow graviton resonances are expected for such small values of $c$.

Tevatron $95 \% \mathrm{CL}$ limits on $M_{G}$ [14] range from $M_{G}>560 \mathrm{GeV}(c=0.01)$ to $M_{G}>1.050 \mathrm{TeV}(c=0.1)$. Thus, due to the high allowed values of $M_{G}$, the discovery could be limited to the lightest $M_{1}$ and the verification of the predicted mass pattern hardly feasible, so that the spin-2 determination through the angular analysis becomes a crucial test of the model.

For dilepton production, the leading order subprocesses $\bar{q} q \rightarrow G \rightarrow l^{+} l^{-}$and $g g \rightarrow$ $G \rightarrow l^{+} l^{-}$give for the $z$-even distributions needed in $A_{\mathrm{CE}}$, with self-explaining notations $[15,16]$ :

$$
\frac{\mathrm{d} \sigma^{G}}{\mathrm{~d} z}=\frac{3}{8}\left(1+z^{2}\right) \sigma_{q}^{\mathrm{SM}}+\frac{5}{8}\left(1-3 z^{2}+4 z^{4}\right) \sigma_{q}^{G}+\frac{5}{8}\left(1-z^{4}\right) \sigma_{g}^{G}
$$

and:

$$
A_{\mathrm{CE}}^{G}=\varepsilon_{q}^{\mathrm{SM}} A_{\mathrm{CE}}^{\mathrm{SM}}+\varepsilon_{q}^{G}\left[2 z^{* 5}+\frac{5}{2} z^{*}\left(1-z^{* 2}\right)-1\right]+\varepsilon_{g}^{G}\left[\frac{1}{2} z^{*}\left(5-z^{* 4}\right)-1\right] .
$$

In (8), $\varepsilon_{q}^{G}, \varepsilon_{g}^{G}$ and $\varepsilon_{q}^{\mathrm{SM}}$ are the fractions of $G$-events under the peak at $M_{R}$ initiated by $\bar{q} q$ and $g g$ processes, and the SM background, respectively. They are determined by overlaps of parton distribution functions and, obviously, $\varepsilon_{q}^{G}+\varepsilon_{g}^{G}+\varepsilon_{q}^{\mathrm{SM}}=1$. Strictly, Eqs. (7) and (8) are quite transparent in showing the characteristic $z$ (and $z^{*}$ ) dependencies for the spin-2 graviton, but hold in that form only for $z_{\text {cut }}=1$. This fact turns out to be numerically unimportant at the optimal value of $z^{*}$ where the $A_{\mathrm{CE}}$ analysis is performed, and the final results take into account all phase space cuts foreseen by the experiment. Moreover, next-to-leading order terms in QCD [17] have for simplicity been included in the calculations through flat (in $z$ ) $K$-factors, $K \simeq 1.3$.

For diphoton production (1), retaining only the leading order RS resonance exchange contributions to simplify the presentation, $\bar{q} q \rightarrow G \rightarrow \gamma \gamma$ and $g g \rightarrow G \rightarrow \gamma \gamma$, the analogues of Eqs. (7) and (8) with the same significance of the notations can be written as [18]:

$$
\frac{\mathrm{d} \sigma^{G}}{\mathrm{~d} z}=\frac{5}{8}\left(1-z^{4}\right) \sigma_{q}^{G}+\frac{5}{32}\left(1+6 z^{2}+z^{4}\right) \sigma_{g}^{G},
$$

and

$$
A_{\mathrm{CE}}^{G}=\varepsilon_{q}^{G}\left[\frac{1}{2} z^{*}\left(5-z^{* 4}\right)-1\right]+\varepsilon_{g}^{G}\left[-1+\frac{5}{8} z^{*}+\frac{5}{4} z^{* 3}+\frac{1}{8} z^{* 5}\right] .
$$

It turns out that in this case the $g g$-initiated subprocess is the dominant, with a shape peaked at $z= \pm 1$ similar to the SM background but by far overwhelming it for the order $\mathrm{TeV}$ values of $M_{G}$ we here are interested in. Clearly, the interest of the diphoton 
channel is that spin- $1 \not \rightarrow \gamma \gamma$ leaves only the spin- 2 and spin- 0 hypotheses, and that the ratio $\operatorname{BR}(G \rightarrow \gamma \gamma) / \mathrm{BR}\left(G \rightarrow l^{+} l^{-}\right) \simeq 2$ is predicted [15].

A current field of interest is the extension of the simplest RS model described here to the case of SM fermions and gauge bosons also propagating in the full 5-D space, this could provide an approach also to other mass hierarchies such as, for example, the fermion mass hierarchy. A complicated phenomenology then emerges, in which both fermions and gauge bosons are accompanied by high (and very high) mass excitations, see for instance the review in Ref. [19]. The existence of the spin-2 graviton excitations remains, however, a general feature. ${ }^{2}$

\section{Heavy neutral gauge bosons}

Turning to the spin-1 hypothesis, $Z^{\prime}$ models depend, besides the mass $M=M_{Z^{\prime}}$, on left- and right-handed couplings to quarks and leptons. In popular scenarios we refer to, generated by different extended electroweak gauge symmetries, those couplings are fixed theoretically, so that only $M_{Z^{\prime}}$ would remain as a free parameter. This is the case of the $Z_{\chi}^{\prime}, Z_{\psi}^{\prime}, Z_{\eta}^{\prime}, Z_{\mathrm{LR}}^{\prime}, Z_{\mathrm{ALR}}^{\prime}$ models, and the "sequential" $Z_{\mathrm{SSM}}^{\prime}$ model with the same couplings as the SM (details can be found in [2]). Current Tevatron lower limits (95\% $\mathrm{CL}$ ) on these $Z^{\prime}$ masses range from $878 \mathrm{GeV}$ to $1.03 \mathrm{TeV}$, depending on the model [21].

Actually, the leading-order partonic subprocess $\bar{q} q \rightarrow Z^{\prime} \rightarrow l^{+} l^{-}$leads to the same form of the $z$-even angular distribution as the SM, therefore to the same $A_{\mathrm{CE}}$ for all models:

$$
\frac{\mathrm{d} \sigma^{Z^{\prime}}}{\mathrm{d} z}=\frac{3}{8}\left(1+z^{2}\right)\left[\sigma_{q}^{\mathrm{SM}}+\sigma_{q}^{Z^{\prime}}\right]
$$

and

$$
A_{\mathrm{CE}}^{Z^{\prime}} \equiv A_{\mathrm{CE}}^{\mathrm{SM}}=\frac{1}{2} z^{*}\left(z^{* 2}+3\right)-1
$$

Our $A_{\mathrm{CE}}$-based estimates for exclusion of the spin- $1 Z^{\prime}$ hypothesis will, accordingly, have a considerable degree of $Z^{\prime}$ model-independence.

\section{Sneutrino exchange}

The spin- 0 character of the resonant subprocess $\bar{d} d \rightarrow \tilde{v} \rightarrow l^{+} l^{-}$, leading to a peak at $M=M_{\tilde{v}}$, manifests itself in a flat angular distribution [3]:

$$
\begin{aligned}
& \frac{\mathrm{d} \sigma^{\tilde{v}}}{\mathrm{~d} z}=\frac{3}{8}\left(1+z^{2}\right) \sigma_{q}^{\mathrm{SM}}+\frac{1}{2} \sigma_{q}^{\tilde{v}}, \\
& A_{\mathrm{CE}}^{\tilde{v}}=\varepsilon_{q}^{\mathrm{SM}} A_{\mathrm{CE}}^{\mathrm{SM}}+\varepsilon_{q}^{\tilde{v}}\left(2 z^{*}-1\right) .
\end{aligned}
$$

Besides $M_{\tilde{v}}$, the cross section is proportional to the $R$-parity violating product $X=$ $\left(\lambda^{\prime}\right)^{2} B_{l}$ where $B_{l}$ is the sneutrino leptonic branching ratio and $\lambda^{\prime}$ the relevant sneutrino coupling to the $\bar{d} d$ quarks. Current constraints on $X$ are very loose (we may take the range $10^{-5}<X<10^{-1}$ ), and the $95 \%$ CL Tevatron lower limits on $M_{\tilde{V}}$ vary from 397 $\mathrm{GeV}\left(X=10^{-4}\right)$ to $866 \mathrm{GeV}\left(X=10^{-2}\right)[21]$.

\footnotetext{
2 The other one being represented by the spin- 0 , massive, radion needed to stabilize the compactification radius $R_{c}$ [20].
} 

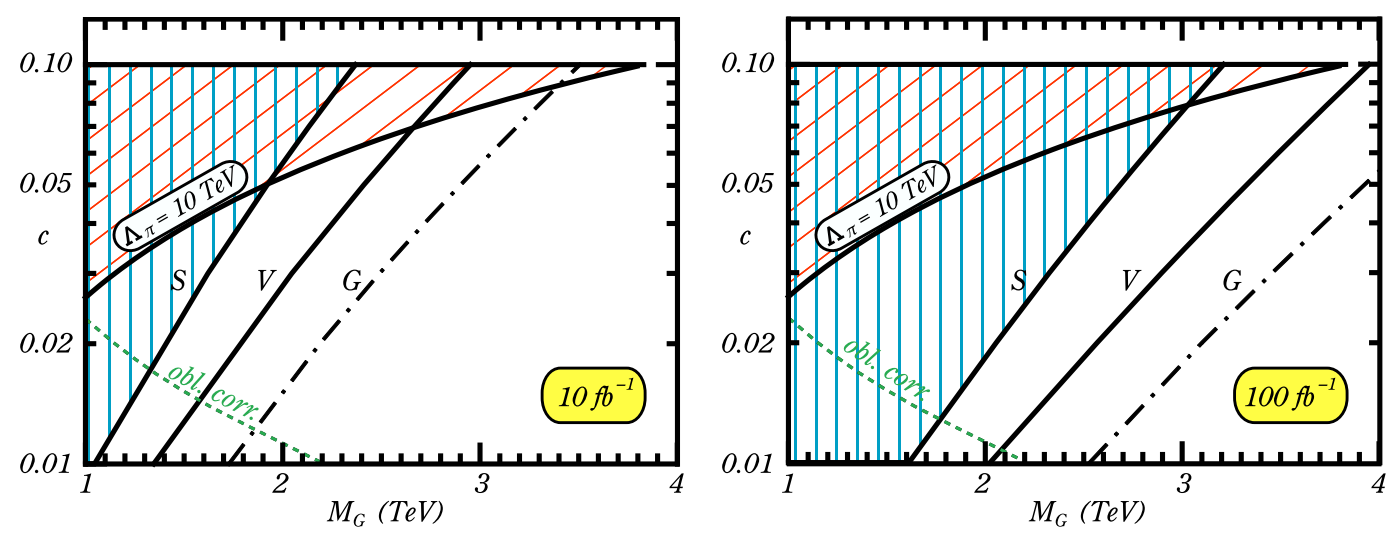

FIGURE 1. Discovery and identification ranges as defined in the text.

\section{RESULTS FOR SPIN-2 IDENTIFICATION}

There are wide domains in $M_{R}$ and coupling constant values allowed by current experimental limits where the scenarios described above predict the same peaks in $M$ with the same numbers of events, so that they cannot be distinguished from each other on the basis of the event rates only.

To proceed with the spin-identifying angular analysis, we suppose that a peak at $M=M_{R}$ is discovered in process (1), and assume that it is consistent with a spin-2 RS graviton (in which case $\left.M_{R} \equiv M_{G}\right)$. To evaluate the domain in the $\left(M_{G}, c\right)$ plane, where the competitor spin-1 and spin-0 hypotheses with the same number of events under the $M=M_{G}$ peak as the graviton hypothesis can be excluded, hence the spin-2 hypothesis can be established, we look at the 'distances' among models:

$$
\Delta A_{\mathrm{CE}}^{Z^{\prime}}=A_{\mathrm{CE}}^{G}-A_{\mathrm{CE}}^{Z^{\prime}} \quad \text { and } \quad \Delta A_{\mathrm{CE}}^{\tilde{v}}=A_{\mathrm{CE}}^{G}-A_{\mathrm{CE}}^{\tilde{v}} .
$$

We adopt a simple-minded $\chi^{2}$-like criterion, where the deviations (15) are compared to the expected statistical uncertainty $\delta A_{\mathrm{CE}}^{G}$ pertinent to the RS model (systematic uncertainties can also be included). The $\chi^{2}$ for the $Z^{\prime}$ and the sneutrino cases in (15) is defined as $\chi^{2}=\left|\Delta A_{\mathrm{CE}}^{Z^{\prime}, \tilde{v}} / \delta A_{\mathrm{CE}}^{G}\right|^{2}$. The desired identification domain in $\left(M_{G}, c\right)$ is determined by the condition $\chi^{2} \geq \chi_{\mathrm{CL}}^{2}$ for both the $Z^{\prime}$ and the $\tilde{v}$ models, with $\chi_{\mathrm{CL}}^{2}$ a critical number that specifies the confidence level (3.84 for 95\% CL). Of course, Eq. (15) depends on the value of $z^{*}$, but it turns out that the choice $z^{*}=0.5$ is 'optimal' in maximizing the sensitivity of the numerical analysis to the RS graviton.

Figure 1 shows the 5- $\sigma$ discovery domains and the 95\% identification domains for LHC luminosities of 10 and $100 \mathrm{fb}^{-1}$ ( $l=e, \mu$ combined), and the 'theoretically favoured' restriction $\Lambda_{\pi}<10 \mathrm{TeV}$ taken into account. Specifically: the area at the left of the line " $G$ " is the discovery domain for the lowest-lying RS graviton resonance; the area at the left of the " $V$ " curve is the exclusion domain of the spin- 1 hypothesis; finally, in the area at the left of the " $S$ " line the spin-0 (and, as one can see, forcefully the spin-1) hypothesis can be excluded. Therefore, the identification domain where both spin-1 and spin-0 hypotheses can be excluded and the spin- 2 established is the intersection of the 
TABLE 1. Discovery and Identification reach $[\mathrm{TeV}]$

\begin{tabular}{lcccc}
\hline & \multicolumn{2}{r}{ Discovery } & \multicolumn{2}{c}{ Identification } \\
\hline $\mathscr{L}_{\text {int }}$ & $c=0.01$ & $c=0.1$ & $c=0.01$ & $c=0.1$ \\
\hline $10 \mathrm{fb}^{-1}$ & $1.7 \mathrm{TeV}$ & $3.5 \mathrm{TeV}$ & $1.1 \mathrm{TeV}$ & $2.4 \mathrm{TeV}$ \\
$100 \mathrm{fb}^{-1}$ & $2.5 \mathrm{TeV}$ & $4.6 \mathrm{TeV}$ & $1.6 \mathrm{TeV}$ & $3.2 \mathrm{TeV}$ \\
\hline
\end{tabular}
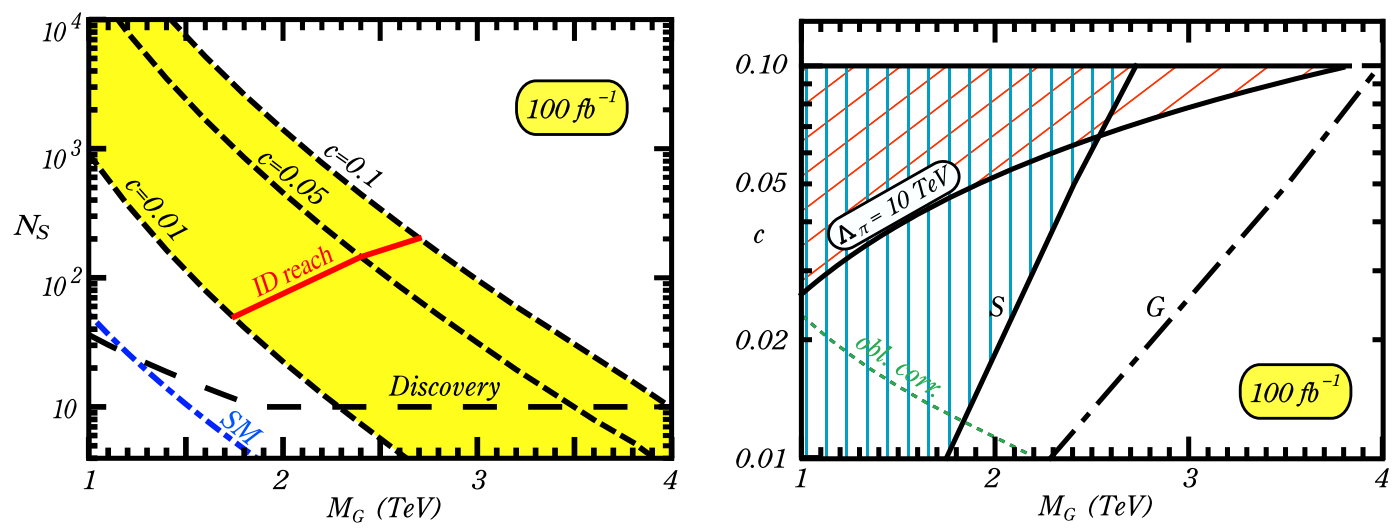

FIGURE 2. Discovery and identification from the process $p p \rightarrow G \rightarrow \gamma \gamma+X$

"S" area with the $\Lambda_{\pi}<10 \mathrm{TeV}$ one, i.e., above that curve. The allowed domain to the right of the dashed "oblique corrections" line is qualitatively determined by a fit to the oblique EW parameters $[13,22]$, but the condition dramatically restricting the discovery domains to the dashed areas, if literally applied, is the $\Lambda_{\pi}$ bound. This condition essentially forbids the creation of additional mass scale hierarchies in the model. Table 1 summarizes the above results obtainable from the observation of RS graviton peaks in dilepton final states.

Figure 2 shows some preliminary attempts to assess both discovery and identification reaches on the spin-2 RS graviton from the diphoton production channel in (1). Specifically, the left panel shows, for $0.01<c<0.1$ the minimum number of events needed to identify by means of the asymmetry $A_{\mathrm{CE}}$ a peak at diphoton invariant mass $M_{G}$ as an RS resonance, against the spin-0 hypothesis (95\% CL and LHC luminosity of $\left.100 \mathrm{fb}^{-1}\right)$. Leading order QCD (therefore unit $K$-factors) has been used to obtain this figure. Also, the foreseen phase space experimental cuts for this channel have been taken into account: $p_{\perp}>40 \mathrm{GeV}$ and $|\eta|<2.4$ for both photons, and reconstruction efficiency $80 \%$. The right panel of Fig. 2 represents, in the same style as Fig. 1, the translation to the $\left(M_{G}, c\right) \mathrm{RS}$ parameter plane of the minimum number of diphoton events for RS graviton identification given in the left panel. This channel is still under study, in particular as regards the effect of the next-to-leading order QCD effects and the perspectives at lower LHC integrated luminosity. Nevertheless, the example in the figure indicates that diphoton events may have a comparable identification sensitivity to the spin-2 RS resonance, with the spin-1 hypothesis automatically excluded. 


\section{ACKNOWLEDGMENTS}

AP and AT acknowledge the partial support of the Belarusian Republican Foundation for Fundamental Research. NP thanks the Organisers for the warm hospitality at a very stimulating and successful Workshop.

\section{REFERENCES}

1. $\quad$ L. Randall and R. Sundrum, Phys. Rev. Lett. 83, 3370 (1999); Phys. Rev. Lett. 83, 4690 (1999).

2. For details and a review see, for example: P. Langacker, Rev. Mod. Phys. 81, 1199 (2008).

3. J. Kalinowski, R. Ruckl, H. Spiesberger and P. M. Zerwas, Phys. Lett. B 406, 314 (1997) 314; 414, 297 (1997).

For a review see: R. Barbier et al., Phys. Rept. 420, 1 (2005).

4. ATLAS Collaboration, Reports No. CERN-LHCC-99-14, CERN-LHCC-99-15.

5. R. Cousins, J. Mumford and V. Valuev [CMS Collaboration], Czech. J. Phys. 55, B651 (2005).

6. J. Pumplin, et al., JHEP 0207, 012 (2002).

7. B. C. Allanach, K. Odagiri, M. A. Parker and B. R. Webber,, JHEP 0009, 019 (2000);

B. C. Allanach, K. Odagiri, M. J. Palmer, M. A. Parker, A. Sabetfakhri and B. R. Webber, JHEP 0212, 039 (2002).

8. R. Cousins, J. Mumford, J. Tucker and V. Valuev, JHEP 0511, 046 (2005).

9. E. W. Dvergsnes, P. Osland, A. A. Pankov and N. Paver, Phys. Rev. D 69, 115001 (2004).

10. P. Osland, A. A. Pankov, N. Paver and A. V. Tsytrinov, Phys. Rev. D 78, 035008 (2008); Phys. Rev. D 79, 115021 (2009).

11. R. Diener, S. Godfrey and T. A. W. Martin, Phys. Rev. D 80, 075014 (2009).

12. H. Murayama and V. Rentala, arXiv:0904.4561 [hep-ph].

13. H. Davoudiasl, J. L. Hewett and T. G. Rizzo, Phys. Rev. Lett. 84, 2080 (2000); Phys. Rev. D 63, 075004 (2001).

14. V. M. Abazov et al. [The D0 Collaboration], Phys. Rev. Lett. 104, 241802 (2010).

15. T. Han, J. D. Lykken and R. J. Zhang, Phys. Rev. D 59, 105006 (1999).

16. G. F. Giudice, R. Rattazzi and J. D. Wells, Nucl. Phys. B 544, 3 (1999).

17. P. Mathews, V. Ravindran and K. Sridhar, JHEP 0510, 031 (2005);

P. Mathews and V. Ravindran, Nucl. Phys. B 753, 1 (2006);

M. C. Kumar, P. Mathews, V. Ravindran and A. Tripathi, Nucl. Phys. B 818, 28 (2009).

18. K. Sridhar, JHEP 0105, 066 (2001);

A. V. Kisselev, JHEP 0809, 039 (2008).

19. H. Davoudiasl, S. Gopalakrishna, E. Ponton and J. Santiago, New J. Phys. 12, 075011 (2010).

20. W. D. Goldberger and M. B. Wise, Phys. Rev. Lett. 83, 4922 (1999); Phys. Lett. B 475, 275 (2000).

21. T. Aaltonen et al. [CDF Collaboration], Phys. Rev. Lett. 102, 091805 (2009).

22. T. Han, D. Marfatia and R. J. Zhang, Phys. Rev. D 62, 125018 (2000). 